\title{
A 3D analysis of the metal distribution in the compact group of galaxies HCG 31
}

\author{
Sergio Torres-Flores ${ }^{1}$, Claudia Mendes de Oliveira ${ }^{2}$, \\ Mayte Alfaro-Cuello ${ }^{1}$, Eleazar Rodrigo Carrasco ${ }^{3}$, Duilia de Mello ${ }^{4}$ \\ and Philippe Amram ${ }^{5}$ \\ ${ }^{1}$ Departamento de Física, Universidad de La Serena, \\ Av. Cisternas 1200 Norte, La Serena, Chile \\ email: storres@dfuls.cl \\ ${ }^{2}$ Instituto de Astronomia, Geofísica e Ciências Atmosféricas da Universidade de São Paulo, \\ Cidade Universitária, CEP:05508-900, São Paulo, SP, Brazil \\ ${ }^{3}$ Gemini Observatory/AURA, Southern Operations Center, Casilla 603, La Serena, Chile \\ ${ }^{4}$ Catholic University of America, Washington, DC 20064, USA \\ ${ }^{5}$ Aix Marseille Université, CNRS, LAM (Laboratoire d'Astrophysique de Marseille) \\ UMR 7326, 13388, Marseille, France
}

\begin{abstract}
We present new Gemini/GMOS integral field unit observations of the central region of the merging compact group of galaxies HCG 31. Using this data set, we derive the oxygen abundances for the merging galaxies HCG $31 \mathrm{~A}$ and HCG 31C. We found a smooth metallicity gradient between the nuclei of these galaxies, suggesting a mixing of metals between these objects. These results are confirmed by high-resolution Fabry-Perot data, from which we infer that gas is flowing between HCG $31 \mathrm{~A}$ and HCG $31 \mathrm{C}$.
\end{abstract}

\section{Main results}

We obtained GMOS/IFU spectra covering the central region of HCG 31A and HCG 31C. We then used the N2 metallicity calibrator defined by Marino et al. (2013) to derive the oxygen abundance between the merging galaxies HCG 31A and HCG 31C. We found that HCG 31A and HCG 31C display values of $12+\log (\mathrm{O} / \mathrm{H})=8.44 \pm 0.03$ and $12+\log (\mathrm{O} / \mathrm{H})=8.22 \pm 0.03$, respectively, with a smooth metallicity gradient between these galaxies. Using high-resolution archival Fabry-Perot data (Amram et al. 2007), we infer that there is gas flowing between these galaxies, detected by a shift in the radial velocities of the $\mathrm{H} \alpha$ emission lines along the line that joins HCG 31A and HCG 31C. This fact suggests a gas mixing between $\mathrm{HCG} 31 \mathrm{AC}$, which can be responsible in producing the observed metal distribution.

\section{Acknowledgements}

ST-F acknowledges the financial support of FONDECYT through a project "Iniciación en la Investigación", under contract 11121505 and the support of the project CONICYT PAI/ACADEMIA 7912010004. MAC acknowledges the financial support of DIULS, through a "Concurso de Apoyo a Tesis 2013", number PT13146. CMdO acknowledges support from FAPESP and CNPq.

\section{References}

Amram, P., Mendes de Oliveira, C., Plana, H., et al. 2007, A\&AA, 471, 753

Marino, R. A., Rosales-Ortega, F. F., Sánchez, S. F., et al. 2013, A\&\&A, 559A, 114 